\title{
Gegen Kopfschmerzen bei Kindern
}

— Schüler fragen ihre Lehrer immer häufiger nach Kopfschmerztabletten: Diese Beobachtung hat die ehemalige Wiesbadener Gesamtschullehrerin Karin Frisch alarmiert. Um den aus ihrer Sicht "gravierenden Anstieg von Kopfschmerzerkrankungen bei Kindern und Jugendlichen entgegenzuwirken", rief sie zusammen mit Professor Hartmut Göbel, Direktor der Schmerzklinik Kiel, vor rund drei Jahren die „Aktion Mütze Kindheit ohne Kopfzerbrechen“ ins Leben. Der Neurologe und Psychologe Göbel ist überzeugt: „Kopfschmerzen kann man durch Wissen verhindern. Wissen ist die beste Medizin, die beste Prävention."

Mehrere Studien zeigten, dass Kopfschmerzen im Kinder- und Jugendalter tendenziell zunähmen, bestätigt auch der Generalsekretär der Deutschen Migräne- und Kopfschmerzgesellschaft, PD Dr. Charly Gaul. Als Gründe würden unter anderem Multitasking und zu wenig verfügbare Freizeit genannt. „Streit im Freundeskreis ist auch ein Risikofaktor, alles was in Richtung Mobbing geht."

\section{Schadensersatz \\ Arzt zahlt für Lebenserhaltung}

_ Weil er einen unheilbar kranken Mann im Endstadium der Demenz immer weiter künstlich am Leben erhielt, soll ein Arzt dem Sohn Schmerzensgeld zahlen. Das Oberlandesgericht München sprach dem Sohn als Alleinerben $40.000 € \mathrm{zu}$ (Az.: $1 \mathrm{U}$ 454/17). Dieser hatte Schmerzensgeld in Höhe von $100.000 €$ und Schadenersatz für Behandlungskosten in Höhe von gut $50.000 €$ geltend gemacht. Der Arzt habe seinen todkranken Vater ohne ausführliche Beratung mit dem Betreuer am Leben erhalten. Die künstliche Ernährung habe das schwere Leiden seines Vaters nur verlängert. Die Verwendung einer Sonde sah er daher als rechtswidrigen körperlichen Eingriff an. (dpa)
Für die „Aktion Mütze" haben Göbel und Frisch eine Unterrichtseinheit entwickelt, die Lehrer, Schüler und deren Eltern gleichermaßen erreichen soll. Ziel ist es, sie umfassend zu informieren, für die Risiken eines unreflektierten Medikamentengebrauchs zu sensibilisieren, Kopfschmerzen vorzubeugen - und die Ursachen anzugehen. Die Unterrichtseinheit können alle siebten Klassen in Deutschland kostenfrei bekommen, wie Frisch sagt. Das Projekt werde zudem mit einer umfassenden wissenschaftlichen Befragung evaluiert. Mehr als 70.000 Schüler haben inzwischen mit den Unterlagen gearbeitet.

„Alles zu Schnelle, alles zu Viele, alles zu Plötzliche, alles zu Intensive kann MigräneAttacken auslösen", mahnt Göbel. Daher sei Regelmäßigkeit im Alltag wichtig: „Kinder sollten einen gleichmäßigen Tagesablauf haben." Außerdem sollten sie zumindest eine halbe Stunde am Tag mal nichts tun: "Handy weglegen, Hinsetzen, zur Ruhe kommen, Träumen und an gar nichts denken“, rät er. „Wenn man das Kindern erklärt,

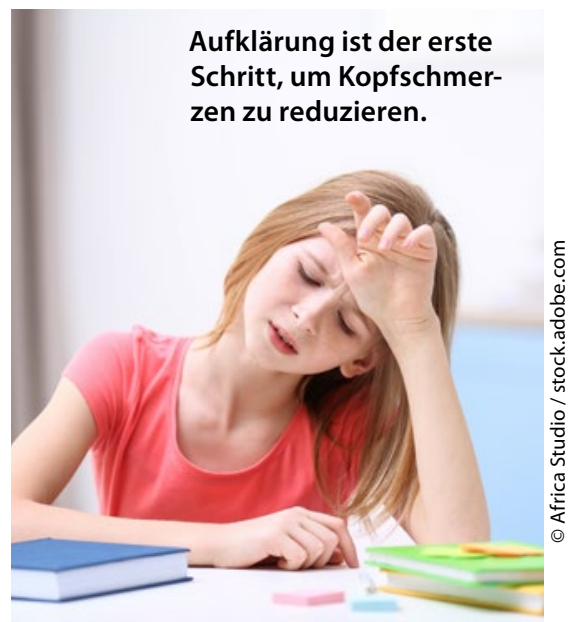

verstehen sie das ganz gut." Hilfreich sei es daher auch Entspannungsverfahren zu lernen. Erste Ergebnisse der "Aktion Mütze" geben den Initiatoren Recht: Am Ende der Erprobungsphase des Projekts hätten mehr als zwei Drittel der befragten Schüler mit Kopfweh angegeben, dass sich ihr Kopfschmerz infolge der Verhaltensumstellung verbessert habe und sie die Unterrichtsmappe immer wieder zurate zögen, sagt Frisch.

(dpa)

\section{Schwangerschaft}

\section{Beeinflusst Paracetamol die kindliche Sprachentwicklung?}

- Die Einnahme von Paracetamol in der Schwangerschaft könnte einer Studie zufolge die Sprachentwicklung des Kindes beeinflussen - allerdings nur bei Mädchen. Forscher um Professor Carl-Gustav Bornehag von der Universität Karlstad untersuchten 754 Frauen in der 8. bis 13. Schwangerschaftswoche [Eur Psych 2018; online 10. Januar].

$60 \%$ gaben an, zwischen Empfängnis und Studienbeginn Paracetamol eingenommen zu haben. Postnatal analysierten die Forscher die Sprachentwicklung der Kinder im Alter von 30 Monaten. Bei Frauen, die im ersten Trimester mehr als sechs Tabletten eingenommen hatten, lag die Wahrscheinlichkeit, dass ihre Töchter eine Sprachverzögerung hatten, fast sechsmal höher als bei Frauen, die kein Paracetamol eingenommen hatten.
Aufgrund der Tatsache, dass die Studienpopulation nur einen kleinen Anteil der ursprünglich rekrutierten Schwangeren ausmacht, interpretieren die Autoren ihr Ergebnis allerdings sehr zurückhaltend. (bae)

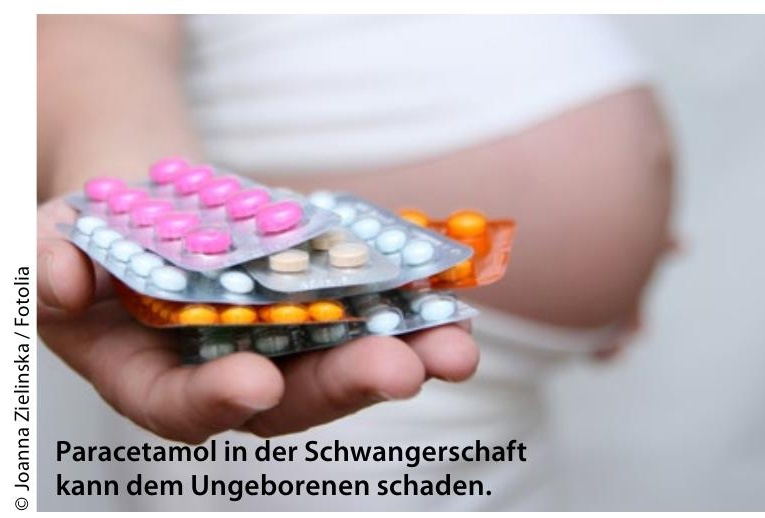

